\title{
Electrophysiological Detection of Delayed Postretinal Neural Conduction in Human Amblyopia
}

\author{
Vincenzo Parisi, ${ }^{1}$ Maria Elisa Scarale, ${ }^{2}$ Nicole Balducci, ${ }^{2}$ Michela Fresina, ${ }^{2}$ \\ and Emilio C. Campos ${ }^{2}$
}

Purpose. To evaluate macular function and neural conduction along postretinal visual pathways in amblyopic patients.

MethoDs. Twenty-five anisometropic amblyopic patients (mean age, $7 \pm 1.9$ years; visual acuity [VA]: $0.44 \pm 0.27$ logMAR in amblyopic $[\mathrm{AM}]$ eyes and $0.023 \pm 0.067 \log \mathrm{MAR}$ in sound $[\mathrm{SE}]$ eyes) and 25 age-similar control subject ([CE] eyes, VA of $0.0 \pm 0.0$ logMAR in both eyes) were enrolled. In AM, SE, and CE eyes, simultaneous pattern electroretinograms (PERGs) and visual evoked potentials (VEPs) were recorded in response to checks reversed at the rate of two reversals/second stimulating macular or extramacular areas (the check edge subtended 15 minutes and 60 minutes of visual arc, respectively).

Results. Nonsignificant differences (ANOVA, $P>0.005$ ) were observed in PERG, in VEP responses to the 60-minute stimulus, and in retinocortical time with the 60-minute stimulus (RCT; the difference between VEP P100 and PERG P50 implicit times) between AM, SE, and CE eyes. AM eyes showed a significant $(P<0.005)$ increase in VEP P100 implicit time and in RCT in response to the 15-minute stimulus, compared with the values observed in SE and CE eyes. In AM patients, the interocular difference in VA was significantly (Pearson's test, $P<0.005)$ related to the interocular difference VEP P100 latencies and RCT with the 15-minute stimulus.

Conclusions. Amblyopic eyes showed abnormal visual cortical responses only when the macular area was stimulated (increase in VEP P100 implicit times with the 15-minute stimulus). This functional impairment, in the presence of normal macular function (PERG responses similar to control eyes) may be attributable to a delay in postretinal neural conduction (increase in RCT). (Invest Ophthalmol Vis Sci. 2010;51: 5041-5048) DOI:10.1167/iovs.10-5412

A mblyopia (AM) is a disorder of the visual system, characterized by reduced visual function in one eye, that has been estimated to affect $1 \%$ to $5 \%$ of the population. ${ }^{1}$ The disorder is associated with strabismus, anisometropia, or a form of deprivation early in life. ${ }^{2-6}$

From the ${ }^{1} \mathrm{G}$. B. Bietti Eye Foundation, IRCCS (Istituto Ricerca e Cura a Carattere Scientifico), Roma, Italy; and the ${ }^{2}$ Ophthalmology Unit, University of Bologna, Bologna, Italy.

Presented at the annual meeting of the Association for Research in Vision and Ophthalmology, Fort Lauderdale, Florida, May 2009.

Supported in part by a grant from "Fondazione Cassa di Risparmio in Bologna" (ECC) and in part by a grant from MIUR (ECC).

Submitted for publication February 21, 2010; revised March 29 and April 22, 2010; accepted April 27, 2010.

Disclosure: V. Parisi, None; M.E. Scarale, None; N. Balducci, None; M. Fresina, None; E.C. Campos, None

Corresponding author: Vincenzo Parisi, G.B. Bietti Eye Foundation, IRCCS, Via Livenza 3, 00199 Roma, Italy; direzionescientifica@fondazionebietti.it.
Several studies have been performed with electrophysiological methods used in humans and in animal models, to investigate the retinal and visual system in AM dysfunction.

Reported findings regarding retinal function are contradictory. The use of electroretinographic signals evoked by flash or patterned stimuli (flash or pattern ERG), which evaluate the function of the outer (preganglionic elements) and the innermost (ganglion cell and their fibers) retinal layers, respectively, ${ }^{7-9}$ showed that AM eyes may present normal and abnormal ERG responses. ${ }^{10-19}$

The function of the entire visual pathway, from photoreceptors to the visual cortex, can be evaluated by visual evoked potential (VEP) recordings, ${ }^{20}$ and the presence of abnormal VEP responses has been observed in amblyopia. ${ }^{21-32}$ Nevertheless, since VEP recordings assess the bioelectrical response of the visual cortex, the observations derived from previous studies $^{21-32}$ do not suggest specific information on whether the reported VEP abnormalities may be selectively related to a retinal dysfunction, a postretinal dysfunction, or both.

That postretinal structures, in particular the lateral geniculate nucleus (LGN), may be involved in AM dysfunctional processes was first suggested by Hubel and Wiesel ${ }^{33}$ and later documented in several studies in which morphologic and functional changes of the LGN were detected. ${ }^{34-40}$

At present, we do not have the means of selectively evaluating the function of the human LGN by electrophysiological methods. However, a particular electrophysiological application consisting of simultaneous recordings of PERG and VEP distinguishes macular from postretinal impairments and provides an electrophysiological index of postretinal neural conduction (derived from the difference between VEP P100 and the PERG P50 implicit times), known as retinocortical time (RCT). ${ }^{41}$

Indeed, RTC was used to suggest a postretinal dysfunction in glaucoma ${ }^{42,43}$ and this hypothesis was later supported by available histologic studies showing that the glaucomatous damage may be related to an impairment occurring in both retinal structures and in the LGN (see Yücel et al. ${ }^{44}$ for a review).

The purpose of our work was to evaluate retinal function and neural conduction along postretinal visual pathways in AM patients by using simultaneous VEP and PERG recordings.

\section{Methods}

\section{Patients}

Twenty-five anisometropic AM patients (mean age, $7.3 \pm 1.9$ years) and 25 age-similar control subjects (mean age, $7.2 \pm 1.6$ years) were enrolled

Since several diseases may induce changes in PERG and VEP responses, for both patients and control subjects the inclusion criteria were the absence of moderate to dense lens opacities, corneal opacities, glaucoma or ocular hypertension, history of intraocular inflamma- 
tion such as anterior or posterior uveitis, history of retinal or macular disease or optic neuropathy, history of ocular trauma, and history of diabetes or other systemic or neurologic diseases

The following methods were used to assess VA and electrophysiological examinations (simultaneous VEP and PERG recordings) in all subjects enrolled.

\section{Best Corrected VA}

Best corrected VA was assessed with the modified Early Treatment Diabetic Retinopathy Study (ETDRS) chart. VA was expressed in logMAR values obtained at a distance of $4 \mathrm{~m}$ with the best refractive correction.

\section{Electrophysiological Examinations}

In agreement with published studies, ${ }^{42,43,45-50}$ simultaneous PERG and VEP recordings were performed with the following methods.

The subject was seated in a semidark, acoustically isolated room in front of the display surrounded by a uniform field of luminance of 5 $\mathrm{cd} / \mathrm{m}^{2}$. Before the experiment, each subject was adapted to the ambient room light for 10 minutes, with a pupil diameter of approximately $5 \mathrm{~mm}$. Mydriatic or miotic drugs were never used. Stimulation was monocular after occlusion of the other eye. Visual stimuli were checkerboard patterns (contrast $80 \%$, mean luminance $110 \mathrm{~cd} / \mathrm{m}^{2}$ ) generated on a TV monitor and reversed in contrast at the rate of two reversals per second. At the viewing distance of $114 \mathrm{~cm}$, the check edges subtended 60 minutes or 15 minutes of visual angle. We used two different checkerboard patterns as suggested by ISCEV standards ${ }^{51}$ to obtain a prevalently macular stimulation (15-minute checks) or a prevalent stimulation of more eccentric retinal areas (60-minute checks). ${ }^{52-54}$ The monitor screen subtended $18^{\circ}$. PERG and VEP recordings were performed with full correction of refraction at the viewing distance. A small, red fixation target, subtending a visual angle of approximately $0.5^{\circ}$ (estimated after taking into account spectaclecorrected individual refractive errors) was placed at the center of the pattern stimulus. At every PERG and VEP examination, each patient positively reported that he/she could clearly perceive the fixation target. The refraction of all subjects was corrected for viewing distance.

PERG Recordings. The bioelectrical signal was recorded by a small Ag/AgCl skin electrode placed over the lower eyelid. PERGs were bipolarly derived between the stimulated (active electrode) and the patched (reference electrode) eye according to a published method. ${ }^{55}$ As the recording protocol was extensive, the use of skin electrodes with interocular recording represented a good compromise between signal-to-noise ratio and signal stability. A discussion on PERGs using skin electrodes and its relationship to the responses obtained by corneal electrodes can be found elsewhere. ${ }^{56,57}$ The ground electrode was in Fpz. ${ }^{58}$ Interelectrode resistance was lower than $3000 \Omega$. The signal was amplified (gain 50,000), filtered (band-pass 1-30 Hz), and averaged, with automatic rejection of artifacts (200 events free from artifacts were averaged for every trial), with an electro-oculography system (BM 6000; Biomedica Mangoni, Pisa, Italy). Analysis time was $250 \mathrm{~ms}$. The transient PERG response is characterized by several waves with three subsequent peaks of negative, positive, and negative polarity, respectively. In visually normal subjects, these peaks have the following implicit times: 35, 50, and $95 \mathrm{~ms}$ (N35, P50, and N95).

VEP Recordings. Cup shaped electrodes of $\mathrm{Ag} / \mathrm{AgCl}$ were fixed with collodion in the following positions: active electrode in $\mathrm{Oz},{ }^{58}$ reference electrode in $\mathrm{Fpz},{ }^{58}$ and ground in the left arm. Interelectrode resistance was kept below $3000 \Omega$. The bioelectric signal was amplified (gain 20,000), filtered (band-pass 1-100 Hz) and averaged (200 events free from artifacts were averaged for every trial) by the electrooculography system (BM 6000; Biomedica Mangoni). Analysis time was $250 \mathrm{~ms}$. The transient VEP response was characterized by several waves with three subsequent peaks of negative, positive, and negative polarity. In visually normal subjects, these peaks have the following implicit times: 75, 100, and $145 \mathrm{~ms}$ (N75, P100, N145).

During a recording session, simultaneous VEPs and PERGs were recorded at least twice (2- 6 times) and the resulting waveforms were superimposed to check the repeatability of results. For all PERGs and VEPs, implicit times and peak-to-peak amplitudes of each of the averaged waves were directly measured on the displayed records by means of a pair of cursors.

On the basis of previous studies (i.e., Parisi et al. ${ }^{49}$ ), we know that intraindividual variability (evaluated by test-retest) is approximately $\pm 2 \mathrm{~ms}$ for PERG P50 and VEP P100 implicit times and approximately $\pm 0.25 \mu \mathrm{V}$ for PERG P50 to N95 and VEP N75 to P100 amplitudes. During the recording session, we considered as superimposable and therefore repeatable two successive waveforms with a difference in milliseconds (for PERG P50 and VEP P100 implicit times) and in microvolts (for PERG P50-N95 and VEP N75-P100 amplitudes), less than the values for intraindividual variability. Sometimes the first two recordings were sufficient to obtain repeatable waveforms; other times, however, further recordings were required (but never $>6$ in the cohort of patients or control subjects). For statistical analyses, we included PERG and VEP values measured in the recording with the lowest PERG P50 to N95 amplitude.

In each subject or patient, the signal-to-noise ratio (SNR) of PERG and VEP responses was assessed by measuring a noise response while the subject fixated an unmodulated field of the same mean luminance as the stimulus. At least two noise records of 200 events each were obtained, and the resulting grand average was used for measurement. The peak-to-peak amplitude of this final waveform (i.e., average of at least two replications) was measured in a temporal window corresponding to that at which the response component of interest (i.e., VEP N75-P100, PERG P50-N95) was expected to peak. SNRs for this component were determined by dividing the peak amplitude of the component by the noise in the corresponding temporal window. An electroretinographic noise $<0.1 \mu \mathrm{V}$ (mean, $0.082 \mu \mathrm{V}$; range, $0.064-0.092 \mu \mathrm{V}$; resulting from the grand average of $400-1200$ events), and an evoked potential noise $<0.15 \mu \mathrm{V}$ (mean, $0.094 \mu \mathrm{V}$; range, 0.075-0.118 $\mu \mathrm{V}$, resulting from the grand average of 400-1200 events) was observed in all subjects tested. In all subjects and patients, we accepted VEP and PERG signals with a signal-to-noise ratio $>2$.

\begin{tabular}{|c|c|c|c|c|c|c|}
\hline & \multirow[b]{2}{*}{ Mean } & \multirow[b]{2}{*}{ SD } & \multicolumn{2}{|c|}{ ANOVA vs. Contralateral } & \multicolumn{2}{|c|}{ ANOVA vs. CE } \\
\hline & & & $\boldsymbol{F}$ & $\boldsymbol{P}$ & $\boldsymbol{F}$ & $\boldsymbol{P}$ \\
\hline CE right eyes & 0 & 0 & & & & \\
\hline CE left eyes & 0 & 0 & $F_{1,49}=0$ & $P=1$ & & \\
\hline $\mathrm{SE}$ & 0.0292 & 0.06757 & & & $F_{1,49}=2.95$ & $P=0.093$ \\
\hline AM & 0.4484 & 0.27521 & $F_{1,49}=54.71$ & $P<0.01$ & $F_{1,49}=66.36$ & $P<0.001$ \\
\hline
\end{tabular}

Data are expressed as mean logMAR VA \pm 1 SD. CE, eyes of control subjects; SE, sound eyes of AM patients; AM, amblyopic eyes of AM patients. 


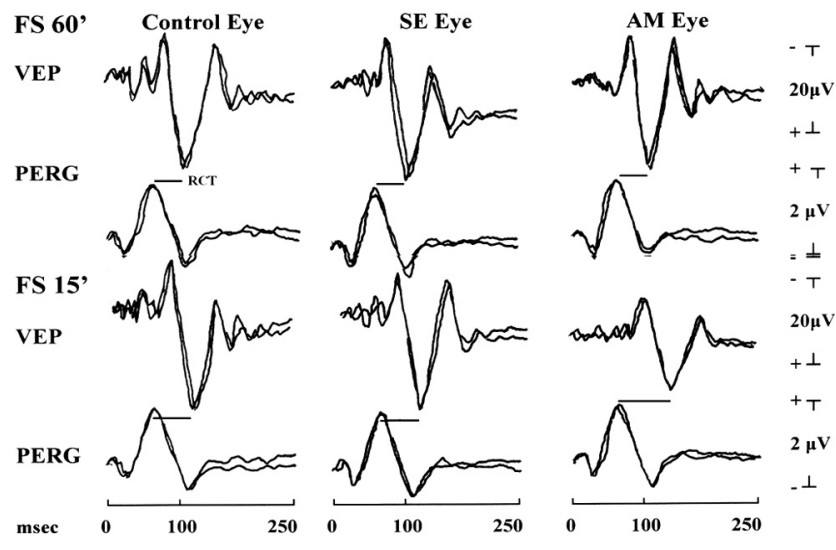

FIGURE 1. Layout of simultaneous recordings of visual evoked potentials (VEPs) and pattern electroretinograms (PERG) in response to a 60or a 15-minute checkerboard stimulus, obtained in one CEs, one SE of an AM patient, and one AM eye of an AM patient. CE, SE, and AM eyes showed similar PERG recordings. VEP and RCT recordings were similar between control and SE eyes with both the 60-minute and 15-minute checks. VEP and RCT recordings were similar between control and AM eyes with 60-minute checks, but with 15-minute checks AM eyes showed a delay in VEP implicit times and a longer RCT with respect to CEs and SEs.

\section{Statistics}

Differences in VA and in PERG and VEP results between groups (control eyes of visually normal subjects, AM eyes, and SEs of AM patients) were evaluated by one-way analysis of variance (ANOVA). Pearson's correlation was used to correlate VA and all electrophysiological parameters (PERG, VEP, and RCT). PERG and VEP implicit times and amplitudes and RCT data underwent logarithmic transformation to better approximate a normal distribution. In all analyses, a conservative $P<0.005$ was considered as statistically significant, to compensate for multiple comparisons $(P=0.05 ; 42$ comparison; $0.05 / 42=0.0012 P$ significance level).

The research followed the tenets of the Declaration of Helsinki and the study was approved by the Local Ethics Committee.

\section{Results}

VA, PERG, and VEP data detected in the AM-affected eyes (25 eyes) of AM patients were separated from sound eyes (SE, 25 eyes) of the same AM patients. These results were considered in the analysis in addition to VA, PERG, and VEP data detected in one eye of normal control subjects (CE, 25 eyes).

\section{Visual Acuity}

Mean values and relative ANOVA results of VA detected in CE, SE, and AM eyes are shown in Table 1. AM eyes showed a significant $(P<0.005)$ reduction in VA when compared with SEs and CEs. No differences $(P>0.005)$ were found between SEs and CEs.

\section{Pattern ERG}

Examples of PERG recordings in one CE, SE, and AM eye are shown in Figure 1. Abnormal PERGs were detected in one SE

TABLE 2. PERG Parameters

\begin{tabular}{|c|c|c|c|c|c|c|}
\hline & \multirow[b]{2}{*}{ Mean } & \multirow[b]{2}{*}{ SD } & \multicolumn{2}{|c|}{ ANOVA vs. Contralateral } & \multicolumn{2}{|c|}{ ANOVA vs. CE } \\
\hline & & & $\boldsymbol{F}$ & $\boldsymbol{P}$ & $\boldsymbol{F}$ & $\boldsymbol{P}$ \\
\hline \multicolumn{7}{|l|}{60 Minute Stimulus } \\
\hline \multicolumn{7}{|l|}{ Control subjects, $n=25$} \\
\hline \multicolumn{7}{|l|}{ Right eyes } \\
\hline PERG P50 IT, ms & 53.96 & 3.34946 & & & & \\
\hline PERG P50-N95 A, $\mu \mathrm{V}$ & 3.1576 & 0.75288 & & & & \\
\hline \multicolumn{7}{|l|}{ Left eyes } \\
\hline PERG P50 IT, ms & 53.4068 & 3.42443 & $F_{149}=0.383$ & $P=0.566$ & & \\
\hline PERG P50-N95 A, $\mu \mathrm{V}$ & 3.0552 & 0.88242 & $F_{1,49}=0.194$ & $P=0.66$ & & \\
\hline \multicolumn{7}{|l|}{ AM patients, $n=25$} \\
\hline \multicolumn{7}{|l|}{$\mathrm{SE}$} \\
\hline PERG P50 IT, ms & 54.3212 & 3.43611 & & & $F_{1,49}=0.888$ & $P=0.351$ \\
\hline PERG P50-N95 A, $\mu \mathrm{V}$ & 3.2496 & 1.30744 & & & $F_{1,49}=0.379$ & $P=0.541$ \\
\hline \multicolumn{7}{|l|}{ AM } \\
\hline PERG P50 IT, ms & 54.0092 & 2.82607 & $F_{1,49}=0.122$ & $P=0.727$ & $F_{1,49}=0.461$ & $P=0.501$ \\
\hline PERG P50-N95 A, $\mu \mathrm{V}$ & 2.9052 & 0.86053 & $F_{1,49}=1.211$ & $P=0.276$ & $F_{1,49}=0.371$ & $P=0.545$ \\
\hline \multicolumn{7}{|l|}{ 15-Minute Stimulus } \\
\hline \multicolumn{7}{|l|}{ Control subjects, $n=25$} \\
\hline \multicolumn{7}{|l|}{ Right eyes } \\
\hline PERG P50 IT, ms & 55.9688 & 3.23995 & & & & \\
\hline PERG P50-N95 A, $\mu \mathrm{V}$ & 3.0356 & 0.71891 & & & & \\
\hline \multicolumn{7}{|l|}{ Left eyes } \\
\hline PERG P50 IT, ms & 55.8612 & 3.1436 & $F_{1,49}=0.014$ & $P=0.905$ & & \\
\hline PERG P50-N95 A, $\mu \mathrm{V}$ & 3.0784 & 0.86804 & $F_{1,49}=0.036$ & $P=0.851$ & & \\
\hline \multicolumn{7}{|l|}{ AM patients, $n=25$} \\
\hline \multicolumn{7}{|l|}{ SE } \\
\hline PERG P50 IT, ms & 55.0708 & 2.78826 & & & $F_{1,49}=0.884$ & $P=0.351$ \\
\hline \multirow{2}{*}{\multicolumn{7}{|c|}{ AM }} \\
\hline & & & & & & \\
\hline PERG P50 IT, ms & 54.4604 & 3.68912 & $F_{1,49}=0.435$ & $P=0.512$ & $F_{1,49}=2.088$ & $P=0.154$ \\
\hline PERG P50-N95 A, $\mu \mathrm{V}$ & 2.6392 & 0.80885 & $F_{1,49}=0.611$ & $P=0.438$ & $F_{1,49}=3.425$ & $P=0.073$ \\
\hline
\end{tabular}

Data are expressed as the mean \pm 1 SD of PERG parameters. CE, eyes of control subjects; SE, sound eyes of AM patients; AM, amblyopic eyes of AM patients; IT, implicit time; A, amplitude. 
and in two AM eyes, when compared with normal limits (mean values of control population -2 SD for $\mathrm{P} 50-\mathrm{N} 95$ amplitude and mean values of control population $+2 \mathrm{SD}$ for $\mathrm{P} 50$ implicit time).

On average, nonsignificant $(P<0.005)$ differences in P50 implicit time and in P50-N95 amplitude were observed between CE, SE, and AM eyes. Mean values and relative ANOVA of PERG results observed in all eyes are reported in Table 2.

In AM eyes, nonsignificant correlations $(P>0.005)$ were found between interocular differences in PERG parameters (P50 implicit time and P50-N95 amplitude) and interocular differences in VA.

\section{Visual Evoked Potentials}

Examples of VEP recordings in one CE, SE, and AM eye are shown in Figure 1. Mean values and relative ANOVA of VEP results observed in $\mathrm{CE}, \mathrm{SE}$, and $\mathrm{AM}$ eyes are reported in Table 3.

Abnormal VEPs (normal limits: mean values of control population -2 SD for N75-P100 amplitude and mean values of control population +2 SD for P100 implicit time) were detected in 2 AM eyes but in none of the SEs with the 60 -minute stimulus. On average, nonsignificant $(P<0.005)$ differences in P100 implicit time and in N75-P100 amplitude were observed between $\mathrm{CE}, \mathrm{SE}$, and AM eyes. Nonsignificant correlations $(P>0.005)$ were found in $\mathrm{CE}, \mathrm{SE}$, and AM eyes between interocular differences in VEP parameters (P100 implicit time and N75 amplitude) and interocular differences in VA.

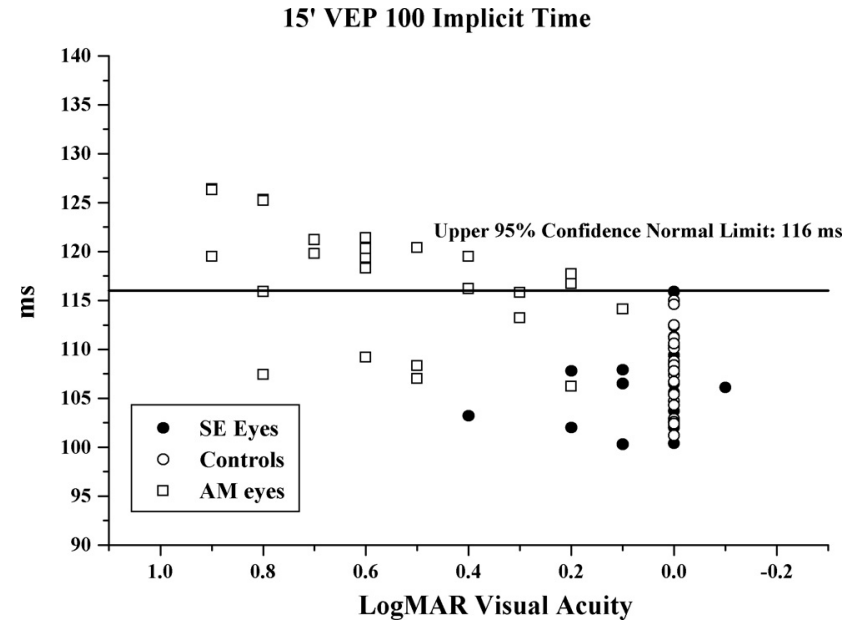

FIGURE 2. Individual values of VEP P100 implicit time recorded in response to 15-minute checks in CEs, in SEs of AM patients (SE), and in AM eyes of AM patients plotted against logMAR VA. Normal limits (solid line) were derived from mean values $+2 \mathrm{SD}$ of the control. In AM eyes, the delay in VEP P100 implicit time approached, but did not reach, statistical significance $(r=-0.4771, P=0.0158$; Pearson's test), when correlated with the decrease in VA.

Abnormal VEPs were detected in 18 AM eyes but in none of the SEs with the 15-minute stimulus. Figure 2 shows individual values of VEP P100 implicit time plotted against

TABLE 3. VEP Parameters: 60-Minute Stimulus

\begin{tabular}{|c|c|c|c|c|c|c|}
\hline & \multirow[b]{2}{*}{ Mean } & \multirow[b]{2}{*}{ SD } & \multicolumn{2}{|c|}{ ANOVA vs. Contralateral } & \multicolumn{2}{|c|}{ ANOVA vs. CE } \\
\hline & & & $\boldsymbol{F}$ & $\boldsymbol{P}$ & $\boldsymbol{F}$ & $\boldsymbol{P}$ \\
\hline \multicolumn{7}{|l|}{60 Minute Stimulus } \\
\hline \multicolumn{7}{|l|}{ Control subjects, $n=25$} \\
\hline \multicolumn{7}{|l|}{ Right eyes } \\
\hline VEP P100 IT, ms & 103.6344 & 4.04758 & & & & \\
\hline VEP N75-P100 A, $\mu \mathrm{V}$ & 21.8304 & 4.38903 & & & & \\
\hline \multicolumn{7}{|l|}{ Left eyes } \\
\hline VEP P100 IT, ms & 103.7368 & 3.523 & $F_{1,49}=0.009$ & $P=0.924$ & & \\
\hline VEP N75-P100 A, $\mu \mathrm{V}$ & 21.7712 & 3.06548 & $F_{1,49}=0.378$ & $P=0.541$ & & \\
\hline \multicolumn{7}{|l|}{ AM patients, $n=25$} \\
\hline \multicolumn{7}{|l|}{ SE } \\
\hline VEP P100 IT, ms & 104.1064 & 3.91847 & & & $F_{1,49}=0.123$ & $P=0.727$ \\
\hline VEP N75-P100 A, $\mu \mathrm{V}$ & 19.1772 & 5.88383 & & & $F_{1,49}=3.821$ & $P=0.057$ \\
\hline \multicolumn{7}{|l|}{ AM } \\
\hline VEP P100 IT, ms & 105.904 & 6.6748 & $F_{1,49}=1.348$ & $P=0.251$ & $F_{1,49}=2.063$ & $P=0.157$ \\
\hline VEP N75-P100 A, $\mu \mathrm{V}$ & 19.7868 & 5.03355 & $F_{1,49}=0.361$ & $P=0.551$ & $F_{1,49}=2.834$ & $P=0.098$ \\
\hline \multicolumn{7}{|l|}{15 Minute Stimulus } \\
\hline \multicolumn{7}{|l|}{ Control subjects, $n=25$} \\
\hline \multicolumn{7}{|l|}{ Right eyes } \\
\hline VEP P100 IT, ms & 107.608 & 3.93689 & & & & \\
\hline VEP N75-P100 A, $\mu \mathrm{V}$ & 21.6032 & 4.24007 & & & & \\
\hline \multicolumn{7}{|l|}{ Left eyes } \\
\hline VEP P100 IT, ms & 107.264 & 3.3354 & $F_{1,49}=0.014$ & $P=0.905$ & & \\
\hline VEP N75-P100 A, $\mu \mathrm{V}$ & 20.9732 & 5.0016 & $F_{1,49}=0.036$ & $P=0.841$ & & \\
\hline \multicolumn{7}{|l|}{ AM patients, $n=25$} \\
\hline \multicolumn{7}{|l|}{ SE } \\
\hline VEP P100 IT, ms & 106.676 & 3.83789 & & & $F_{1,49}=0.334$ & $P=0.565$ \\
\hline VEP N75-P100 A, $\mu \mathrm{V}$ & 17.6672 & 7.50638 & & & $F_{1,49}=0.358$ & $P=0.073$ \\
\hline \multicolumn{7}{|l|}{$\mathrm{AM}$} \\
\hline VEP P100 IT, ms & 117.228 & 6.01723 & $F_{1,49}=54.64$ & $P<0.001$ & $F_{1,49}=52.43$ & $P<0.001$ \\
\hline VEP N75-P100 A, $\mu \mathrm{V}$ & 11.8388 & 5.18313 & $F_{1,49}=10.83$ & $P<0.001$ & $F_{1,49}=72.18$ & $P=0.002$ \\
\hline
\end{tabular}

Data are expressed as the mean \pm 1 SD of VEP parameters. SE, sound eyes of AM patients; AM, amblyopic eyes of AM patients; IT, implicit time; A, amplitude. 

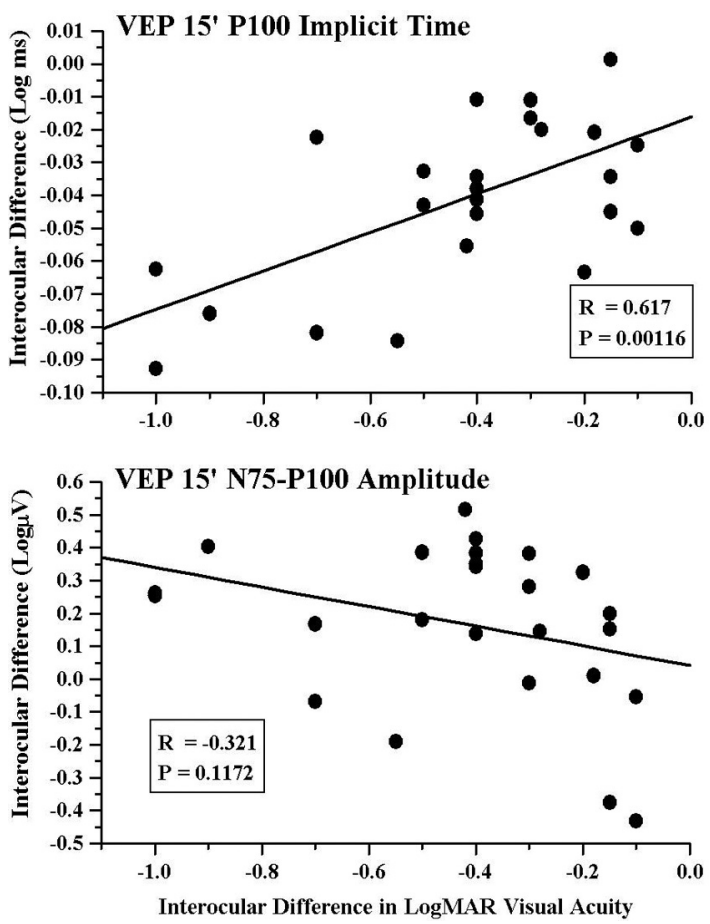

FIGURE 3. Individual interocular differences in VEP P100 implicit time and N75-P100 amplitude recorded in response to 15-minute checks in AM eyes plotted against the interocular difference in logMAR VA. Pearson's test was used for correlation analysis.

relative values of VA observed in $\mathrm{CE}, \mathrm{SE}$, and $\mathrm{AM}$ eyes. In AM eyes, the delay in VEP P100 implicit time approached, but did not reach, statistical significance $(r=-0.4771, P=$ 0.0158 ), when correlation with the decrease in VA was analyzed.

On average, nonsignificant $(P<0.005)$ differences in P100 implicit time and in N75-P100 amplitude were observed between CEs and SEs, whereas AM eyes showed a significant $(P<$ $0.005)$ delay in $P 100$ implicit time and a significant $(P<0.005)$ decrease in N75-P100 amplitude with respect to SEs and CEs.

Nonsignificant correlations $(P>0.005)$ were found in CEs and SEs between interocular differences in VEP parameters (P100 implicit time and N75 amplitude) and interocular differences in VA. A significant correlation $(P<0.005)$ was observed in AM eyes between the interocular difference in VEP P100 implicit time and the interocular difference in VA. Nonsignifi-

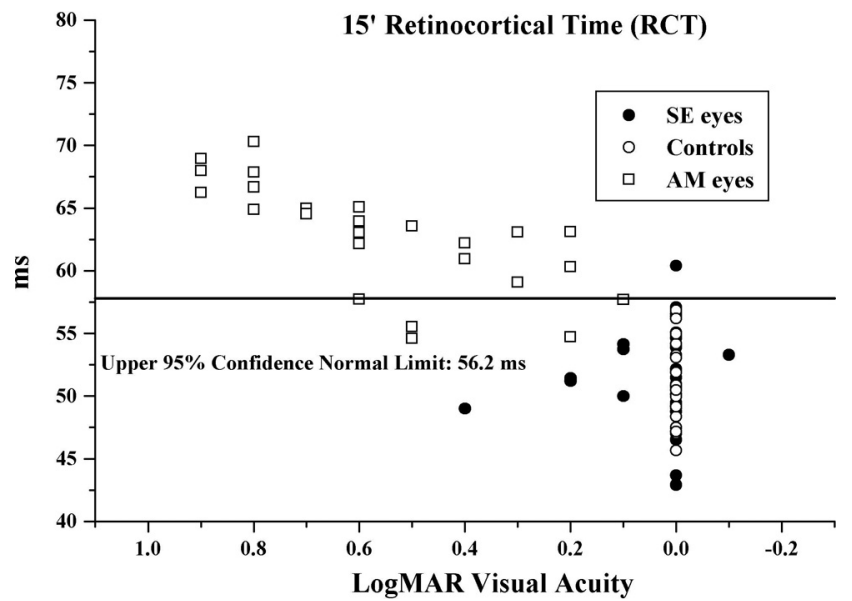

FigURE 4. Individual RCTs recorded in response to 15-minute checks in CEs, in SEs of AM patients, and in AM eyes of AM patients plotted against logMAR VA. Normal limits (solid line) were derived from mean values $+2 \mathrm{SD}$ of control subjects. In AM eyes, the delay in RCT correlated $(r=-0.714, P<0.001$; Pearson's test) with the decrease in VA.

cant correlations $(P>0.005)$ were found between the interocular difference in VEP N75-P100 amplitude and the interocular difference in VA. Individual values and relative correlations are presented in Figure 3.

\section{Retinocortical Time}

Mean values and relative ANOVA of RCT results observed in CE, SE, and AM eyes are shown in Table 4. Abnormal RCTs (normal limits: mean values of control population +2 SD for P100 implicit time) were detected in two AM eyes but in none of the SEs with the 60-minute stimulus. On average, RCT was similar $(P>0.005)$ in CE, SE, and AM eyes. Nonsignificant correlations $(P>0.005)$ were detected in $\mathrm{CE}, \mathrm{SE}$, and AM eyes between the interocular difference in RCT and the interocular difference in VA.

Abnormal RCT was detected in one SE and in 22 AM eyes with the 15 -minute stimulus. Figure 4 shows individual values of RCT plotted against the relative VAs observed in CE, SE, and AM eyes. In AM eyes, the delay in RCT correlated significantly $(r=-0.714, P<0.001)$ with the decrease in VA. On average, nonsignificant $(P>0.005)$ differences in RCT were observed between CEs and SEs, whereas AM eyes showed a significantly $(P<0.005)$ delayed RCT when compared with SEs and CEs.

TABLE 4. RCT Results

\begin{tabular}{|c|c|c|c|c|c|c|}
\hline & \multirow[b]{2}{*}{ Mean } & \multirow[b]{2}{*}{ SD } & \multicolumn{2}{|c|}{ ANOVA vs. Contralateral } & \multicolumn{2}{|c|}{ ANOVA vs. CE } \\
\hline & & & $\boldsymbol{F}$ & $\boldsymbol{P}$ & $\boldsymbol{F}$ & $\boldsymbol{P}$ \\
\hline \multicolumn{7}{|c|}{ 60-Minute stimulus } \\
\hline CE right eyes & 49.6744 & 3.61649 & & & & \\
\hline CE left eyes & 50.33 & 4.14354 & $F_{149}=0.355$ & $P=0.553$ & & \\
\hline SE & 49.7852 & 5.14341 & & & $F_{1,49}=1.701$ & $P=0.681$ \\
\hline AM & 51.8948 & 7.11344 & $F_{1,49}=1.443$ & $P=0.235$ & $F_{1,49)}=0.903$ & $P=0.346$ \\
\hline \multicolumn{7}{|c|}{ 15-Minute stimulus } \\
\hline CE right eyes & 51.6392 & 3.29326 & & & & \\
\hline CE left eyes & 51.4028 & 3.51418 & $F_{1,49}=0.013$ & $P=0.908$ & & \\
\hline SE & 51.6052 & 3.9202 & & & $F_{1,49}=0.036$ & $P=0.848$ \\
\hline $\mathrm{AM}$ & 62.7676 & 4.34975 & $F_{1,49}=90.84$ & $P<0.001$ & $F_{1,49}=103.26$ & $P<0.001$ \\
\hline
\end{tabular}

RCT is the difference between VEP P100 and PERG P50 implicit times. Data are expressed as the mean RCT \pm 1 SD. CE, eyes of control subjects; SE, sound eyes of AM patients; AM, amblyopic eyes of AM patients. 


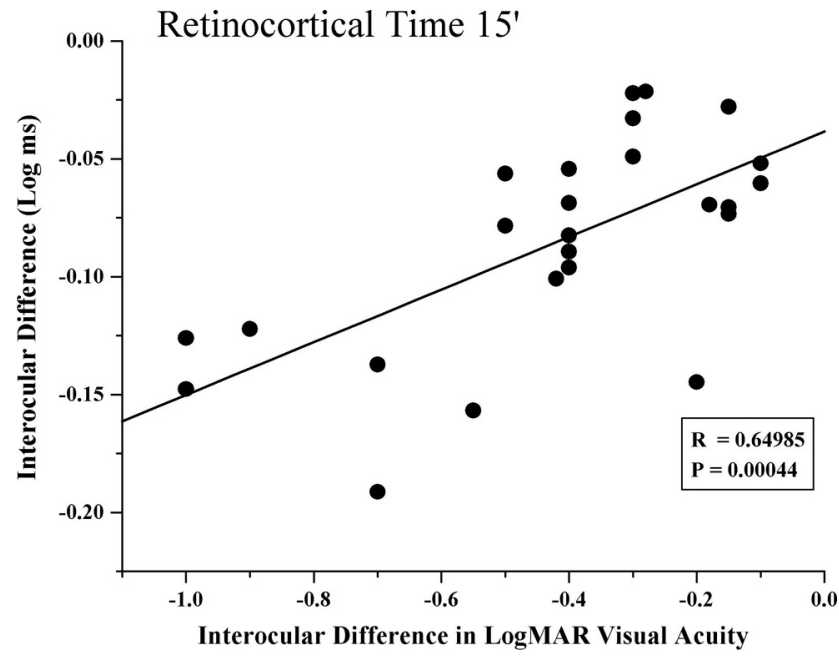

FIGURE 5. Individual values of interocular difference in RCTs recorded in response to a 15-minute checkerboard in AM eyes plotted against the interocular difference in logMAR VA. In AM eyes, the interocular difference in RCT correlated with the interocular difference in VA. Pearson's test was used for correlation analysis.

Nonsignificant correlations $(P>0.005)$ were found in CEs and SEs between the interocular difference in RCT and the interocular difference in VA. A significant correlation $(P<$ 0.005 ) was found between the interocular difference in RCT and the interocular difference in VA of AM eyes. Individual values and relative correlations are presented in Figure 5.

\section{Discussion}

The purpose of our work was to evaluate retinal function and neural conduction along postretinal visual pathways in AM patients by using an electrophysiological approach (simultaneous PERG and VEP recordings).

\section{Retinal Function: PERG data}

PERG parameters (P50 implicit time and P50-N95 amplitude) evaluate the bioelectrical responses of different retinal elements. In fact, a contribution of preganglionic elements has been suggested in the genesis of the P50 component, ${ }^{59}$ and the integrity of the innermost retinal layers (ganglion cell and their fibers) is necessary to obtain a normal PERG P50-N95 amplitude. ${ }^{59-62}$

PERG implicit times and amplitudes were not significantly different in the group of AM patients when compared with control subjects, and this suggests, in agreement with previous studies, ${ }^{16-19}$ that retinal function is similar in anisometropic AM patients and visually normal subjects. In agreement with this hypothesis, no morphologic retinal changes have been identified by the nerve fiber analysis (GDx NFA; Carl Zeiss Meditec, Oberkochen, Germany) in patients with anisometropic and strabismic amblyopia. ${ }^{63}$ By contrast, other studies show abnormal ERG responses, suggesting a retinal dysfunction, in human or animal amblyopia. ${ }^{10-15}$

\section{Visual Cortical Responses: VEP Data}

In our electrophysiological protocol, according to ISCEV standards, ${ }^{51}$ we used two sizes of checkerboard patterns: 60- and 15-minute.

Findings in several studies suggest that high spatial frequencies (i.e., 15-minute checks) preferentially stimulate the macular region, which has a much larger cortical representation, as opposed to lower spatial frequencies (i.e., 60-minute checks), which preferentially stimulate more eccentric (peripheral or extramacular) retinal areas. ${ }^{52,53}$ An interesting study performed in patients with macular hole has shown that 15minute checks stimulate the macular region and that VEPs in response to checks larger than 15 minutes are independent from the macula and may therefore indicate the cortical bioelectrical activity in response to the stimulation of more eccentric retinal areas. ${ }^{54}$ Indeed, abnormal VEP responses were detected in these patients only when high spatial frequencies (i.e., 17 minutes) were used. On the contrary, VEPs recorded in response to larger checks (i.e., 34 minutes) showed normal responses notwithstanding the presence of a macular hole in the patients. ${ }^{54}$

It is well known that different visual stimuli activate different components of the visual pathways. In fact, when large (60-minute) checks are used in visually normal subjects, VEPs with shorter implicit times are obtained than those in response to small (15-minute) checks. ${ }^{61}$ Thus, a visual stimulus consisting of large checks may activate large receptive retinal fields located more peripherally with respect to the central retina, and the bioelectrical signal is driven to the visual cortex by large axons (with a fast neural conduction), which constitute the prevalence of the magnocellular system. By contrast, when small checks (15 minutes) are used, smaller receptive retinal fields located in the central retina are probably activated and, in this case, the bioelectrical signal is driven to the visual cortex by small axons (with a reduced speed in neural conduction), which constitute the prevalence of the parvocellular system. $^{64}$

Our AM patients showed abnormal cortical responses (VEP responses with delayed implicit times) only when a macular stimulus (15-minute checks) was used; normal VEP responses were found in the presence of a peripheral stimulus (60-minute checks). That implicit times are prolonged when the central region of the visual field is stimulated is also suggested by results obtained by multifocal VEP recordings in amblyopia. ${ }^{65}$ All this suggests that these visual stimuli, and in particular high spatial frequencies (i.e., 15-minute checks), are appropriate to detect abnormal cortical responses in AM patients.

These results suggest that the magnocellular-dependent visual cortical responses obtained in our AM patients are functionally spared in amblyopia, in the presence of a functional impairment of the parvocellular system. This hypothesis is supported by results obtained by other electrophysiological methods ${ }^{66}$ and by the measurement of blood flow response in the primary visual cortex by positron emission tomography (PET). ${ }^{67}$

\section{Neural Conduction along Postretinal Visual Pathways: RCT Data}

The purpose of our study was also to evaluate neural conduction along postretinal visual pathways by using an electrophysiological index derived from the difference between VEP P100 and PERG P50 implicit times, known as retinocortical time. ${ }^{41}$

Our AM patients, showed an RCT within our normal limits in the presence of normal PERG and normal VEP recordings, in response to large (60-minute) checks. A longer RCT, together with delayed VEP implicit time and normal PERG, was observed when small (15-minute) checks were used. All this suggests that, in AM eyes, abnormal cortical responses are influenced by a delay in postretinal visual pathways and are independent from retinal function, only when the macular region is stimulated.

Results in studies of glaucoma in which PERG and RCT were used $^{42,43}$ suggest the presence of two sources of functional impairment in this pathologic condition: one at the retinal level (abnormal PERG) and one at the level of the LGN 
(delay in RCT). This electrophysiological evidence was later supported by histologic studies. ${ }^{44}$

RCT cannot be used to selectively differentiate a single structural dysfunction of the postretinal visual pathways (i.e., optic chiasm, LGN, optic radiation) ${ }^{43}$; yet, an explanation regarding the delay in RCT in AM patients may be offered by the available data reporting the effects of amblyopia at the LGN level.

In fact, histologic studies have shown that morphologic alterations are present at the level of the LGN in experimental animal models of amblyopia as well as in humans with amblyopia. In particular, the cells of the parvocellular system of the LGN receiving signals from the AM eye are smaller and more weakly colored than are normal eye cells. A functional loss of these cells may be due to an improper stimulation of the macular area by unclear images during the critical phase of visual development. It is not yet clear whether LGN alterations occur as primary damage or as a consequence of modification of the visual cortex, which could affect the LGN through a retrograde process. ${ }^{33-40}$

Electrophysiological data (abnormal RCT), obtained with small (15-minute checks) visual stimuli, confirm a possible impairment in the parvocellular system of the LGN in amblyopia. Our results are in agreement with those in another study. ${ }^{19}$ However, although simultaneous PERG and VEP recordings were performed in a preceding study, ${ }^{10}$ RCT was not evaluated. Therefore, apart from the data proposed by Teping et al., ${ }^{19}$ there is no further information in the literature regarding postretinal neural conduction in AM patients.

In humans, the presence of different RCTs between the AM and the sound eye could be the electrophysiological evidence of imbalanced inputs coming from the AM and sound retina to the LGN and consequently to the visual cortex. This imbalance in turn could result in the neurophysiologic mechanism that is at the basis of visual plasticity and that has been well described, beginning with the study by Hubel and Wiesel. ${ }^{33}$ The significant correlation between interocular RCT and VA differences suggests that the development of different visual acuities in the $\mathrm{AM}$ and in the sound eye may depend on these imbalanced neural conductions.

In conclusion, our results suggest that abnormal cortical responses (delayed VEPs) in AM patients can be ascribed to a delayed neural conduction in postretinal visual pathways (longer RCT) with concomitant presence of normal retinal function (normal PERG).

\section{References}

1. Flom MC, Neumaier RW. Prevalence of amblyopia. Public Health Rep. 1966;81:329-341.

2. Birch EE. Infant interocular acuity differences and binocular vision. Vision Res. 1985;25:571-576.

3. Eggers HM, Blakemore C. Physiological basis of anisometropic amblyopia. Science. 1978;201:264-267.

4. Harwerth RS, Smith EL, Boltz RL, Crawford MLJ, von Noorden GK. Behavioral studies on the effects of abnormal early visual experience in monkeys: spatial modulation sensitivity. Vision Res. 1983; 23:1501-1510.

5. Hess RF, Holliday IE. The spatial localization deficit in amblyopia. Vision Res. 1992;32:1319-1339.

6. Von Noorden GK. Classification of amblyopia. Am J Ophthalmol. 1967;63:238-244.

7. Maffei L, Fiorentini A. Electroretinographic responses to alternating gratings before and after section of the optic nerve. Science. 1981;211:953-955.

8. Maffei L, Fiorentini A. Electroretinographic responses to alternating gratings in the cat. Exp Brian Res. 1982;48:327-334.

9. Morrone MC, Fiorentini A, Bisti S, Porciatti V, Burr DC. Patternreversal electroretinogram in response to chromatic stimuli. II. Monkey. Vis Neurosci. 1994;11:873-884.
10. Sokol S, Nadler D. Simultaneous electroretinograms and visually evoked potentials from adult amblyopes in response to a pattern stimulus. Invest Ophthalmol Vis Sci. 1979;18:848-855.

11. Persson HE, Wanger P. Pattern-reversal electroretinograms in squint amblyopia, artificial anisometropia and simulated eccentric fixation. Acta Ophthalmol. 1982;60:123-132.

12. Arden GB, Wooding SL. Pattern ERG in amblyopia. Invest Ophthalmol Vis Sci. 1985;26:88-96.

13. Arden GB, Vaegan, Hogg CR, Powell DJ, Carter RM. Pattern ERGs are abnormal in many amblyopes. Trans Ophthalmol Soc. 1980; 100:453-460.

14. Ikeda $\mathrm{H}$, Tremain KE. Amblyopia occurs in retinal ganglion cells in cats reared with convergent squint without alternating fixation. Exp Brain Res. 1979;35:559-582.

15. Dahlke C, Dodt E. Amblyopic eyes produce an abnormal electroretinogram in pattern presentation with the on-off technique. Ophthalmologe. 1994;91:176-180.

16. Yin ZQ, Li CY, Pei X, Vaegan, Fang QX. Development of pattern ERG and pattern VEP spatial resolution in kittens with unilateral esotropia. Invest Ophthalmol Vis Sci. 1994;35:626-634.

17. Gottlob I, Welge-Lüssen L. Normal pattern electroretinograms in amblyopia. Invest Ophthalmol Vis Sci. 1987;28:187-191.

18. Hess RF, Baker CL Jr, Verhoeve JN, Keesey UT, France TD. The pattern evoked electroretinogram: its variability in normals and its relationship to amblyopia. Invest Ophthalmol Vis Sci. 1985;26: $1610-1623$

19. Teping C, Kamps I, Reim M. Retinal and retinocortical times to pattern stimulation in amblyopic children. Doc Ophthalmol. 1989; 73:111-117.

20. Celesia GG, Bodis-Wollner I, Chatrian GE, Harding GFA, Sokol S, Spekreijse H. Recommended standards for electroretinograms and visual evoked potentials: report of an IFCN Committee. Electroenceph Clin Neurophysiol. 1993;87:421-436.

21. Johansson B, Jakobsson P. Fourier-analysed steady-state VEPs in pre-school children with and without normal binocularity. Doc Ophthalmol. 2006;112:13-22.

22. Oner A, Coskun M, Evereklioglu C, Dogan H. Pattern VEP is a useful technique in monitoring the effectiveness of occlusion therapy in amblyopic eyes under occlusion therapy. Doc Ophthalmol. 2004; 109:223-227.

23. Davis AR, Sloper JJ, Neveu MM, Hogg CR, Morgan MJ, Holder GE. Electrophysiological and psychophysical differences between early- and late-onset strabismic amblyopia. Invest Ophthalmol Vis Sci. 2003;44:610-617.

24. Basmak H, Yildirim N, Erdinç O, Yurdakul S, Ozdemir G. Effect of levodopa therapy on visual evoked potentials and visual acuity in amblyopia Ophthalmologica. 1999;213:110-113.

25. Porciatti V, Schiavi C, Benedetti P, Baldi A, Campos EC. Cytidine-5 minute-diphosphocholine improves visual acuity, contrast sensitivity and visually-evoked potentials of amblyopic subjects. Curr Eye Res. 1998;17:141-148.

26. Davis ET, Bass SJ, Sherman J. Flash visual evoked potential (VEP) in amblyopia and optic nerve disease. Optom Vis Sci. 1995;72:612618.

27. Ridder WH 3rd, Rouse MW. Predicting potential acuities in amblyopes: predicting post-therapy acuity in amblyopes. $D o c O p h$ thalmol. 2007;114:135-145.

28. Henc-Petrinović L, Deban N, Gabrić N, Petrinović J. Prognostic value of visual evoked responses in childhood amblyopia. Eur J Ophthalmol. 1993;3:114-120.

29. Beneish R, Lachapelle P, Polomeno R, Lake N. Pattern VEP differences in strabismic and anisometric amblyopia. Clin Vision Sci. 1990;5:271-283.

30. Campos EC, Prampolini ML, Gulli R. Contrast sensitivity differences between strabismic and anisometropic amblyopia: objective correlate by means of visual evoked responses. Doc Ophthalmol. 1984;58:45-50.

31. Sokol S, Hansen VC, Moskowitz A, Greenfield P, Towle VL. Evoked potential and preferential looking estimates of visual acuity in pediatric patients. Ophthalmology. 1983;90:552-562.

32. Sokol S. Abnormal evoked potential latencies in amblyopia. $\mathrm{BrJ}$ Ophthalmol. 1983;67:310-314. 
33. Hubel DH, Wiesel TN. Binocular interaction in striate cortex of kittens reared with artificial squint. J Neurophysiol. 1965;28:10411059.

34. Hess RF, Thompson B, Gole G, Mullen KT. Deficient responses from the lateral geniculate nucleus in humans with amblyopia. Eur J Neurosci. 2009;29:1064-1070.

35. Miki A, Liu GT, Goldsmith ZG, Liu CS, Haselgrove JC. Decreased activation of the lateral geniculate nucleus in a patient with anisometropic amblyopia demonstrated by functional magnetic resonance imaging. Ophthalmologica. 2003;217:365-369.

36. Cheng G, Kaminski HJ, Gong B, et al. Monocular visual deprivation in macaque monkeys: a profile in the gene expression of lateral geniculate nucleus by laser captures microdissection. Mol Vis. 2008;4:1401-1413.

37. Carmignoto G, Canella R, Candeo P, Comelli MC, Maffei L. Effects of nerve growth factor on neuronal plasticity of the kitten visual cortex. J Physiol. 1993;464:343-360.

38. von Noorden GK, Crawford ML. The lateral geniculate nucleus in human strabismic amblyopia. Invest Ophthalmol Vis Sci. 1992;33: $2729-2732$.

39. von Noorden GK, Crawford ML, Levacy RA. The lateral geniculate nucleus in human anisometropic amblyopia. Invest Ophthalmol Vis Sci. 1983;24:788-790.

40. Berardi N, Domenici L, Parisi V, Pizzorusso T, Cellerino A, Maffei L. Monocular deprivation effects in the rat visual cortex and lateral geniculate nucleus are prevented by nerve growth factor (NGF). I. Visual cortex. Proc Biol Sci. 1993;251:17-23.

41. Celesia GG, Kaufmann D. Pattern ERG and visual evoked potentials in maculopathies and optic nerve disease. Invest Ophthalmol Vis Sci. 1985;26:726-735.

42. Parisi V. Neural conduction in the visual pathways in ocular hypertension and glaucoma. Graefes Arch Clin Exp Ophthalmol. 1997;235:136-142.

43. Parisi V. Impaired visual function in glaucoma. Clin Neurophysiol. 2001;112:351-358.

44. Yücel YH, Zhang Q, Weinreb RN, Kaufman PL, Gupta N. Effects of retinal ganglion cell loss on magno-, parvo-, koniocellular pathways in the lateral geniculate nucleus and visual cortex in glaucoma. Prog Retin Eye Res. 2003;22:465-481.

45. Parisi V, Manni G, Spadaro M, et al. Correlation between morphological and functional retinal impairment in multiple sclerosis patients previously affected by optic neuritis. Invest Ophthalmol Vis Sci. 1999;40:2520-2528.

46. Parisi V, Manni GL, Gandolfi SA, et al. Visual function correlates with nerve fiber layer thickness in eyes affected by ocular hypertension. Invest Ophthalmol Vis Sci. 1999;40:1828-1833.

47. Parisi V, Manni GL, Colacino G, Bucci MG. Cytidine-5 minutediphosphocholine (Citicoline) improves retinal and cortical responses in patients with glaucoma. Ophthalmology. 1999;106: $1126-1134$

48. Parisi V, Manni G, Centofanti M, et al. Correlation between optical coherence tomography, pattern electroretinogram and visual evoked potentials in open angle glaucoma patients. Ophthalmology. 2001;108:905-912.

49. Parisi V, Miglior S, Manni G, Centofanti M, Bucci MG. Clinical ability of pattern electroretinograms and visual evoked potentials in detecting visual dysfunction in ocular hypertension and glaucoma. Ophthalmology. 2006;113:216-228.

50. Parisi V, Gallinaro G, Ziccardi L, Coppola G. Electrophysiological assessment of visual function in patients with non-arteritic ischaemic optic neuropathy. Eur J Neurol. 2008;15:839-845.

51. Odom JV, Bach M, Brigell M, et al. ISCEV standard for clinical visual evoked potentials (2009 update). Doc Ophthalmol. 2010;120:111119.

52. Harter MR, White CT. Evoked cortical responses to checkerboard patterns: effect of check-size as a function of visual acuity. Electroencephalogr Clin Neurophysiol. 1970;28:48-54.

53. Celesia GG. Evoked potential techniques in the evaluation of visual function. J Clin Neurophysiolol. 1984;1:55-76.

54. Tilanus MA, Cuypers MH, Bemelmans NA, Pinckers AJ, Deutman $\mathrm{AF}$. Predictive value of pattern VEP, pattern ERG and hole size in macular hole surgery. Graefes Arch Clin Exp Ophthalmol. 1999; 237:629-635.

55. Fiorentini A, Maffei L, Pirchio M, Spinelli D, Porciatti V. The ERG in response to alternating gratings in patients with diseases of the peripheral visual pathway. Invest Ophthalmol Vis Sci. 1981;21: $490-493$.

56. Hawlina M, Konec B. New non-corneal HK-loop electrode for clinical electroretinography. Doc Ophthalmol. 1992;81:253-259.

57. Porciatti V, Falsini B. Inner retina contribution to the flicker electroretinogram: a comparison with the pattern electroretinogram. Clin Vision Sci. 1993;8:435-447.

58. Jasper HH. The ten-twenty electrode system of the international federation of electroencephalography. Electronceph Clin Neurophysiol. 1958;10:371-375.

59. Viswanathan S, Frishman LJ, Robson JG. The uniform field and pattern ERG in macaques with experimental glaucoma: removal of spiking activity. Invest Ophthalmol Vis Sci. 2000;41:2797-2810.

60. Parisi V, Restuccia R, Fattapposta F, et al. Morphological and functional retinal impairment in Alzheimer's disease patients. Clin Neurophysiol. 2001;112:1860-1867.

61. Holder GE. The pattern electroretinogram in anterior visual pathways dysfunction and its relationship to the pattern visual evoked potential: a personal clinical review of 743 eyes. Eye. 1997;11: 924-934.

62. Harrison JM, O'Connor PS, Young RS, Kincaid M, Bentley R. The pattern ERG in man following surgical resection of the optic nerve. Invest Ophthalmol Vis Sci. 1993;28:492- 499.

63. Bozkurt B, Irkeç M, Orhan M, Karaağaoğlu E. Thickness of the retinal nerve fiber layer in patients with anisometropic and strabismic amblyopia. Strabismus. 2003;11:1-7.

64. Jones R, Keck MJ. Visual evoked response as a function of grating spatial frequency. Invest Ophthalmol Vis Sci. 1978;17:652-659.

65. Yu M, Brown B, Edwards MH. Investigation of multifocal visual evoked potential in anisometropic and esotropic amblyopes. Invest Ophthalmol Vis Sci. 1998;39:2033-2040.

66. Shan Y, Moster ML, Roemer RA, Siegfried JB. Abnormal function of the parvocellular visual system in anisometropic amblyopia. $J P e$ diatr Ophthalmol Strabismus. 2000;37:73-78.

67. Mizoguchi S, Suzuki Y, Kiyosawa M, Mochizuki M, Ishii K. Differential activation of cerebral blood flow by stimulating amblyopic and fellow eye. Graefes Arch Clin Exp Ophthalmol. 2005;243: 576-582. 